\title{
Urinary Frequency
}

National Cancer Institute

\section{Source}

National Cancer Institute. Urinary Frequency. NCI Thesaurus. Code C26906.

Urination at short intervals; it may result from increased urine formation, decreased bladder capacity, or lower urinary tract irritation. 